Review Article

\title{
Progress on the Experimental Research of Sciatic Nerve Injury with Acupuncture
}

\author{
Hui Wang, ${ }^{1,2}$ Jingjing Cui, ${ }^{1}$ Shitong Zhao, ${ }^{3}$ Dongsheng Xu, ${ }^{1}$ Shuang Wu, ${ }^{1}$ Wanzhu Bai $\mathbb{D}^{1},{ }^{1}$ \\ and Jia Wang $\mathbb{B}^{1}$ \\ ${ }^{1}$ Institute of Acupuncture and Moxibustion, China Academy of Chinese Medical Sciences, Beijing 100700, China \\ ${ }^{2}$ Center for Experimental Medicine, The First Affiliated Hospital of Nanchang University, Nanchang, Jiangxi 330006, China \\ ${ }^{3}$ Department of Traditional Chinese Medicine, Beijing Chaoyang Hospital, Capital Medical University, Beijing, China
}

Correspondence should be addressed to Wanzhu Bai; wanzhubaisy@hotmail.com and Jia Wang; wangjia315500@163.com

Received 12 August 2021; Accepted 3 December 2021; Published 23 December 2021

Academic Editor: Amjad Iqbal

Copyright (c) 2021 Hui Wang et al. This is an open access article distributed under the Creative Commons Attribution License, which permits unrestricted use, distribution, and reproduction in any medium, provided the original work is properly cited.

Objective. To collect and summarize relevant literatures on the experimental researches of sciatic nerve injury (SNI) with acupuncture during the last decade providing a guideline for effectively treating SNI with acupuncture in the future. Methods. The Chinese and English databases including China National Knowledge Infrastructure (CNKI), Wanfang Data Knowledge Service Platform (WanFang Data), VIP Information Chinese Journal Service Platform (VIP Date), and PubMed were searched from 2009 to 2020 with keywords of "acupuncture and moxibustion OR acupuncture OR electroacupuncture OR scalp acupuncture OR wrist-ankle acupuncture OR acupoint injection OR ear acupuncture" AND "sciatic nerve OR sciatic nerve injury OR sciatic injury OR SNI." The collected data were mainly evaluated in the items of animal model of SNI, type of interventions, selection of acupuncture points (acupoints), course of treatment and its frequency, and approaches of assessment. Results. A total of 89 studies were included in this analysis. Among them, the most commonly used animal models of SNI were produced by the clamp or transverse injury in the rats; the most frequently used intervention was electroacupuncture with dilatational wave of $2 / 100 \mathrm{~Hz}$; the frequency of acupuncture was mainly performed once per day lasting for more than 2 weeks; the mainly selected acupoints were Huantiao (GB30), Zusanli (ST36), and Yanglingquan (GB34); and the approaches of assessment were contained with behavioral, functional, morphological, histological, cellular, and molecular measurements. Conclusion. The results indicated that the experimental researches of SNI with acupuncture has made marked progress in recent years, which may provide important clues for further investigating the underlying mechanisms of acupuncture for the treatment of SNI in the future.

\section{Introduction}

Sciatic nerve injury (SNI), as a typical type of peripheral nerve injury, is a common disorder to meet the acupuncture treatment [1-4]. Usually, SNI might be caused by incidents such as trauma, crush injury, sharp instrument attacking, drug injection, pelvic fracture, and hip dislocation, manifesting with sensory dysfunction such as pain, numbness, and loss of sensation, as well as locomotor dysfunction such as muscular atrophy, muscular tone decreasing, and limb paralysis [1, 5-7]. Although SNI is not lethal, it can cause long-term functional deficits in patients $[8,9]$.
Up to now, in order to treat SNI, many efforts have been made in clinical practices, including the transplantation of neural tissues and stem cells, the administration of neurotrophic medicine and nerve growth factor, as well as physical and laser therapies $[10,11]$. Although all of them had certain curative effects on the SNI, considering their side effects and relatively high costs, some of the patients also accepted acupuncture as a complementary or alternative choice and obtained satisfied effects from treatment [12-14]. However, the underlying mechanism of acupuncture for meliorating SNI still remains unclear. 
In order to tackle this problem, a large number of experimental researches on SNI with acupuncture has been carried out and reported separately in the last decade [15-17]. In this study, we collected the relevant literatures in this field from the Chinese and English databases with correlated keywords and evaluated them in the items of the animal model of SNI, type of interventions, selection of acupuncture points (acupoints), course of treatment and its frequency, and approaches of assessment, respectively. By analyzing these data, we expect to summarize the progress on the experimental research of SNI with acupuncture and provide a clue for further investigating its underlying mechanism associated with the clinical application.

\section{Materials and Methods}

\subsection{Criteria for Study Inclusion and Exclusion}

(1) Type of studies: the original studies related to the experimental research of acupuncture for SNI were included. However, the clinical trials, experience introductions, reviews, and other relevant literatures would be excluded.

(2) Type of research object: rat, rabbit, mouse, cat, monkey, dog, etc., no limitation on animal species and gender.

(3) Type of intervention: any type of acupuncture was included, including acupuncture, electroacupuncture, scalp acupuncture, ear acupuncture, wrist-ankle acupuncture, and acupoint injections.

(4) Types of outcomes: the animal model of SNI, type of interventions, selection of acupoints, course of treatment and its frequency, and approaches of assessment were mainly analyzed.

2.2. Search Strategy. Taking "acupuncture and moxibustion OR acupuncture OR electroacupuncture OR scalp acupuncture OR wrist-ankle acupuncture OR ear acupuncture OR acupoint injection" AND "sciatic nerve OR sciatic nerve injury OR sciatic injury OR SNI" as the keywords searched for the literature related to acupuncture intervention for SNI on the Chinese and English databases, including China National Knowledge Infrastructure (CNKI, 2009-2020), Wanfang Data Knowledge Service Platform (WanFang Data, 2009-2020), VIP Information Chinese Journal Service Platform (VIP Date, 2009-2020), and PubMed (2009-2020).

2.3. Data Extraction and Analysis. Two reviewers (Hui Wang and Jia Wang) independently used a predesigned data extraction form for rigorous data collection, including general information such as authors of studies, year of publication, and animal model of SNI, as well as treatment information including type of intervention, selection of acupoints, course of treatment and its frequency, and approaches of assessment. Finally, it would be systematically summarized and analyzed.

\section{Results}

3.1. Study Selection. Through electronic searching, 10795, 13746, and 8562 records in Chinese were identified from CNKI, Wanfang, and VIP Date databases, respectively, and 166 records in English were found from PubMed. By eliminating studies that were irrelevant, repetitive, and unable to obtain the full text, there were totally 89 studies included in accordance with the inclusion and exclusion criteria. Among these, there were 83 studies published in Chinese and 6 studies in English.

\subsection{Characteristics of Included Studies}

3.2.1. Annual Publication. During the past twelve years (2009-2020), there has been an overall upward trend in the number of annual publications on acupuncture treatment for SNI. Counting by the number of publications in a twoyear interval, it was observed that the highest number of publications appeared in 2019-2020 with 29 studies, followed by 2015-2016 with 22 studies (Figure 1).

3.2.2. Animal Species. In the 89 included studies, the animals used for preparing the SNI model included rats, mice, and rabbits. Among them, the rat was by far the most commonly employed animal model in SNI experimental research, with the rabbit being the second most popular model, accounting for $84.3 \%$ (75/89) and $14.6 \%(13 / 89)$, respectively. There was only one study that applied to the mouse (Figure 2).

3.2.3. Animal Model of SNI. By summarizing the included studies, the animal models of SNI were established by many methods, including transverse, clamp, ligation, or injection injury to sciatic nerve [18-21]. Among them, the model with clamp injury was the most frequently used, and the model with transverse injury was the second most applied, with 61 $(61 / 89,68.5 \%)$ and 19 studies $(19 / 89,21.3 \%)$, respectively.

\subsection{Application Characteristics of Acupuncture on SNI}

3.3.1. Type of Interventions. Among the 89 included studies, the types of interventions mainly included electroacupuncture, manual acupuncture, ankle acupuncture, and scalp acupuncture, accounting for $93.3 \%$ (83/89), 4.5\% $(4 / 89), 1.1 \%(1 / 89)$, and $1.1 \%(1 / 89)$, respectively (Figure 3$)$. In some of the included studies, multiple interventions of acupuncture were simultaneously used.

In the 83 studies of electroacupuncture, besides 7 studies that did not clearly indicate the stimulated parameters, the other 76 studies marked the definite stimulated styles, in which the waveforms of electroacupuncture were used orderly with dilatational wave $(64.5 \%, 49 / 76)$, sparse wave $(19.7 \%, 15 / 76)$, continuous wave $(11.8 \%, 9 / 76)$, and intermittent wave $(3.9 \%, 3 / 76)$. The frequencies of electroacupuncture were applied orderly with $2 / 100 \mathrm{~Hz}(64.5 \%$, $49 / 76),<10 \mathrm{~Hz}(19.7 \%, 15 / 76), 15 \mathrm{~Hz}(9.2 \%, 7 / 76)$, and 


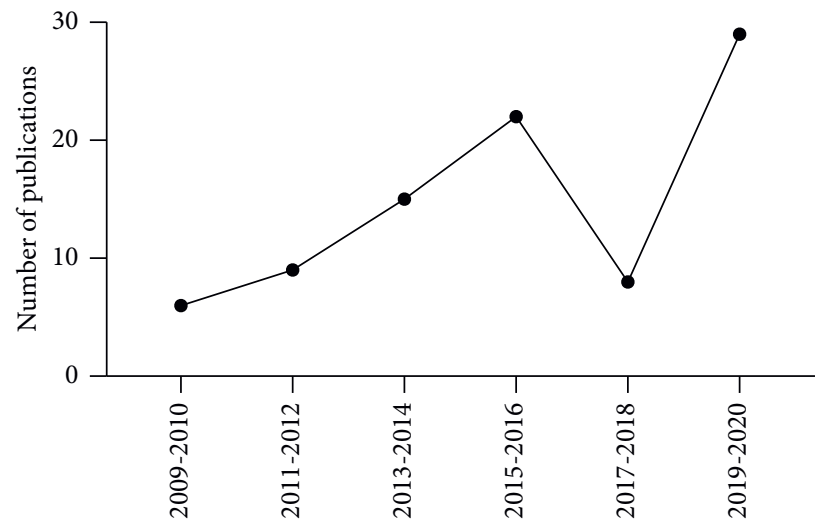

Figure 1: Publication status of experimental research of SNI with acupuncture during 2009-2020.

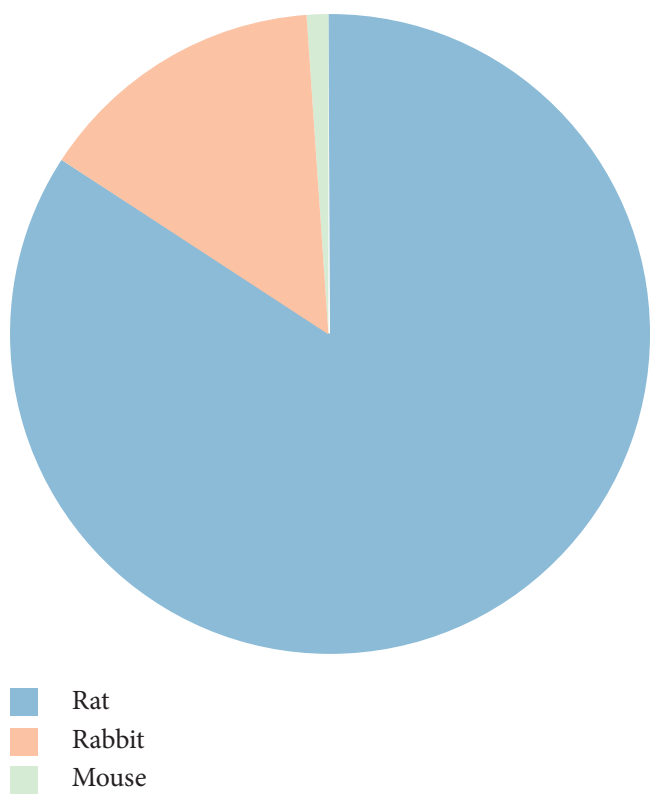

FIgURE 2: Animal application status in experimental research of SNI with acupuncture.

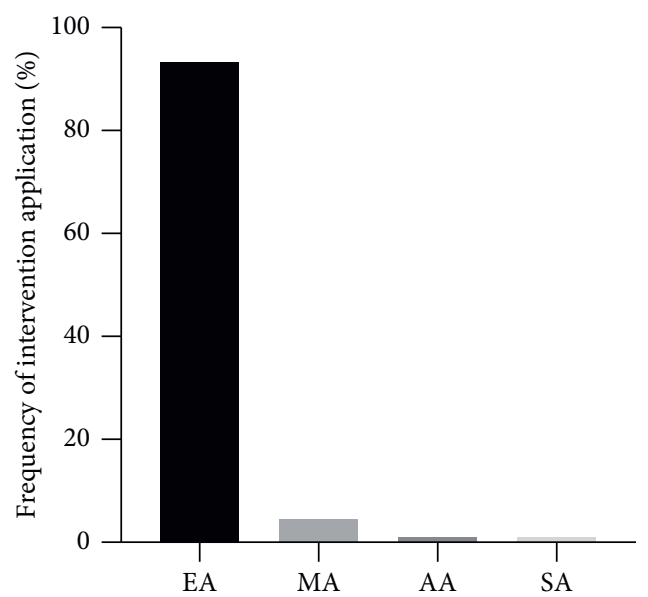

FIgURE 3: The intervention application status in experimental research of SNI with acupuncture. Note. EA: electroacupuncture; MA: manual acupuncture; AA: ankle acupuncture; SA: scalp acupuncture. 


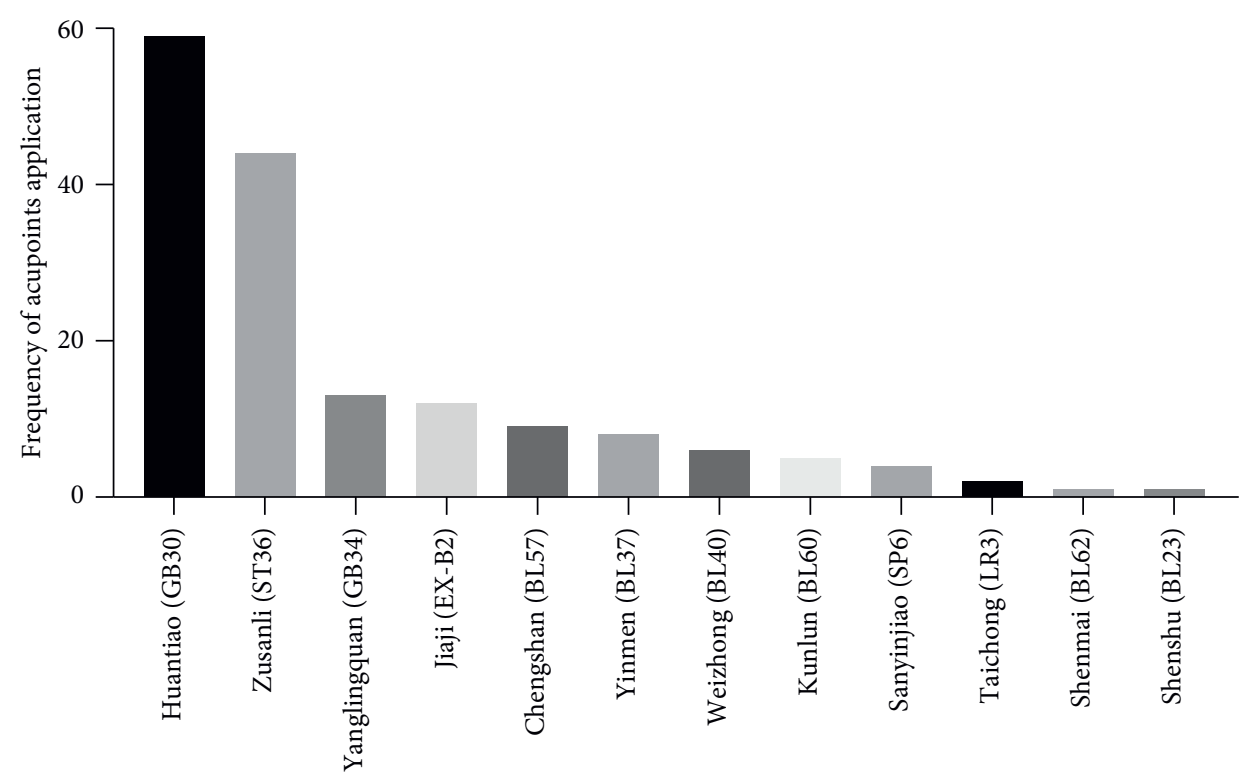

FIGURE 4: The acupoint selection in experimental research of SNI with acupuncture.

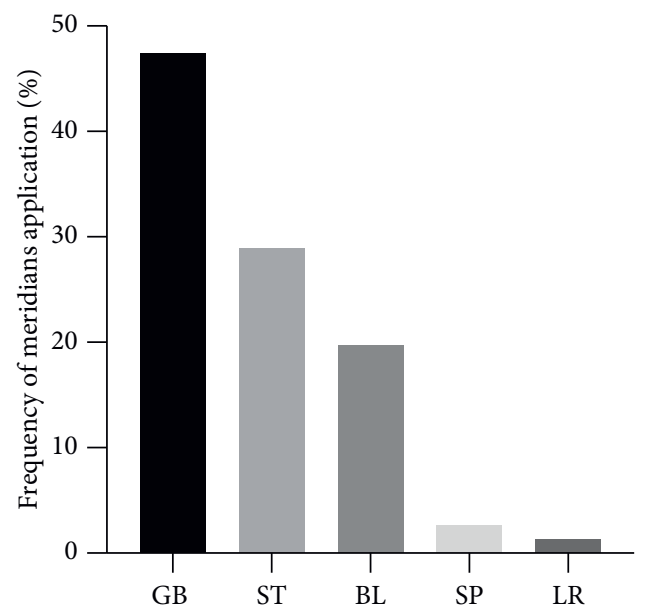

FIgURE 5: The meridians selection in experimental research of SNI with acupuncture. Note. GB: gallbladder meridian; ST: stomach meridian; BL: bladder meridian; SP: spleen meridian; LR: liver meridian.

$20 \mathrm{~Hz}(6.6 \%, 5 / 76)$, and the electric current and voltage were used to cause mild muscle tremors $(84.2 \%, 64 / 76), 1.5 \mathrm{~mA}$ (3.9\%, 3/76), $2 \mathrm{~mA}(3.9 \%, 3 / 76), 20 \mathrm{mV}$ (3.9\%, 3/76), and $1.5 \mathrm{~V}(3.9 \%, 3 / 76)$. In addition, the time of treatment was varied from $9 \mathrm{~min}$ to $30 \mathrm{~min}$ in 80 studies in order of $20 \mathrm{~min}$ $(36.25 \%, 29 / 80), 10 \mathrm{~min}(31.25 \%, 25 / 80), 15 \mathrm{~min}(25.0 \%$, $20 / 80)$, $30 \mathrm{~min}(3.75 \%, 3 / 80)$, and $9 \min (3.75 \%, 3 / 80)$.

3.3.2. Selection of Acupoints. A total of 13 acupoints appeared in the 89 studies, in which Huantiao (GB30, 59/89, 66.3\%) and Zusanli (ST36, 44/89, 49.4\%) appeared most frequently, followed with Yanglingquan (GB34, 13/89, 14.6\%) and Jiaji (EX-B2, 12/89, 13.5\%) (Figure 4).

Except Jiaji, the other acupoints mainly belong to the 5 meridians of the gallbladder (GB), stomach (ST), bladder (BL), spleen (SP), and liver (LR) meridians (Figure 5).
3.3.3. Treatment Frequency and Course. In the included studies, only one study did not mention the frequency of acupuncture. Among the other 88 studies, most studies applied the frequency of once per day $(86 / 88,97.7 \%)$. Only 2 studies applied once in every 2 days $(2 / 88,2.3 \%)$.

Regarding the treatment course, many studies performed acupuncture for more than 2 weeks (56.2\%, 50/89), while another 33 studies performed for $1-2$ weeks $(37.1 \%$, $33 / 83)$, and 6 studies performed for less than 1 week $(6.7 \%$, $6 / 89)$.

\subsection{Analysis Methods Related to SNI with Acupuncture}

3.4.1. Behavioral Measurement. Among the total included studies, 52 studies applied the behavioral measurements, including sciatic nerve function index (SFI), pain threshold, 


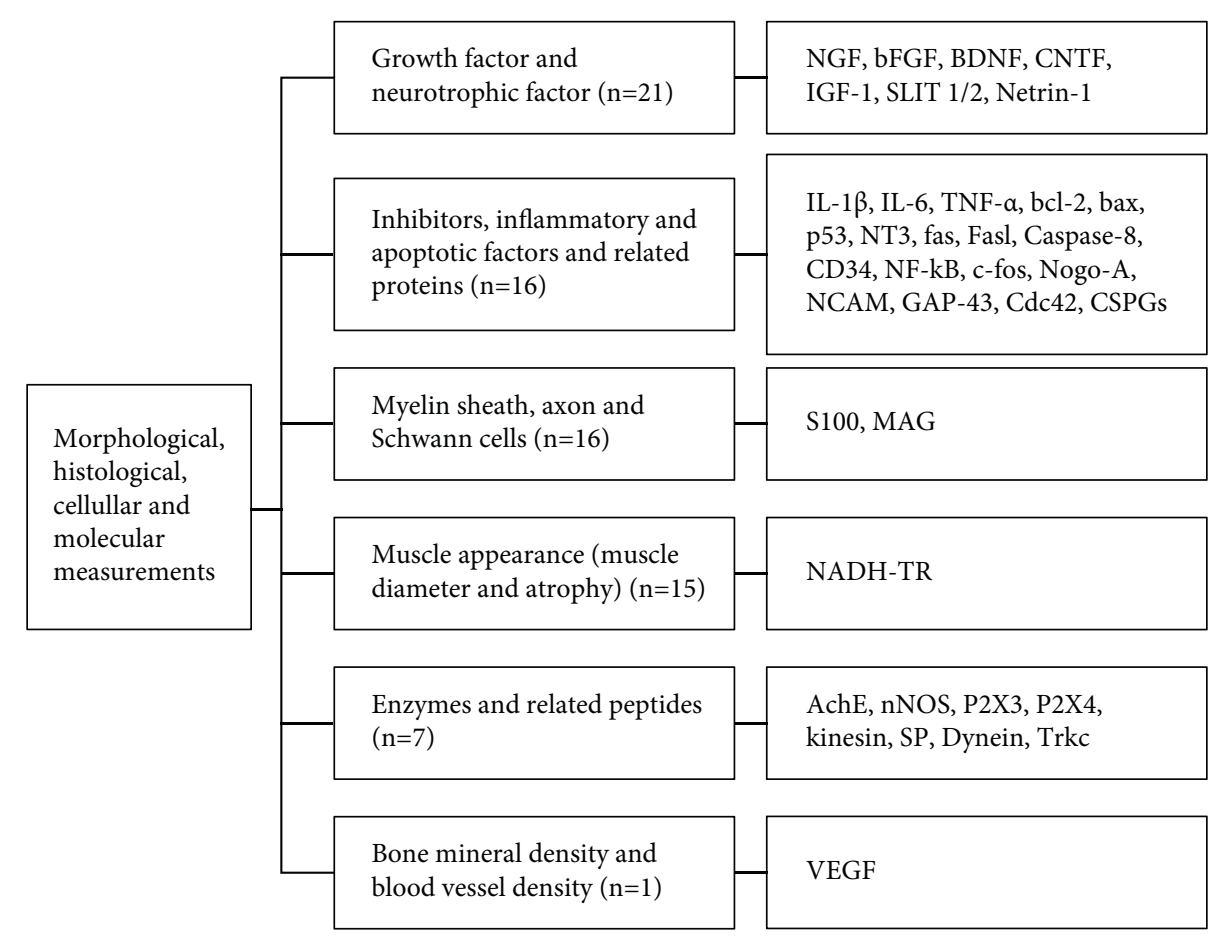

FIGURE 6: The detailed measurements and indicators in the experimental research of SNI with acupuncture. Note: AchE: acetylcholinesterase; bcl-2: b cell lymphoma-2; BDNF: brain-derived neurotrophic factor; bFGF: basic fibroblast growth factor; Cdc42: cell division cycle 42; CNTF: ciliary neurotrophic factor; CSPGs: chondroitin sulfate proteoglycans; GAP-43: growth-associated protein-43; IGF-1: insulin-like growth factor 1; IL-1 $\beta$ : interleukin-1 beta; IL-6: interleukin 6; MAG: myelin-associated glycoprotein; NADH-TR: nicotinomide adenine dinucleotide dehydrogenase-tetrazolium reductase; NCAM: neural cell adhesion molecule; NF-kB: nuclear factor kB; NGF: nerve growth factor; NT3: neurotrophin-3; nNOS: neuronal nitric oxide synthase; SLIT 1/2: slit guidance ligand 1/2; SP: substance P; TNF- $\alpha$ : tumor necrosis factor- $\alpha$; Trkc: tropomyosin receptor kinase C; VEGF: vascular endothelial growth factor.

toe abduction reflex, and inclined pulling test. Among them, SFI was the most widely used $(57.7 \%, 30 / 52)$, followed by the measurement of thermal pain threshold $(30.8 \%, 16 / 52)$. In addition, $15.6 \%(5 / 52)$ and $1.9 \%(1 / 52)$ of studies applied the toe abduction reflex and inclined pulling test, respectively.

3.4.2. Functional Assessment. There were 33 studies that applied the functional assessment, including nerve and muscle function assessments. Among them, 30 studies mainly measured the sciatic nerve function as well as sensory and motor nerve conduction velocity. Another 3 studies measured electromyography and muscle contractility.

\subsubsection{Morphological, Histological, Cellular, and Molecular} Measurements. The approaches of assessments related to the effects and mechanisms of SNI with acupuncture mainly included morphological, histological, cellular, and molecular measurements. There were 58 studies that applied morphological and histological testing, and 44 studies used cellular and molecular testing. The spinal cord, dorsal root ganglia (DRG), sciatic nerve and its related muscles were the main tissues to be examined. The growth factor and neurotrophic factor were the most frequently tested indicators, with 21 studies applied. Besides, inhibitors inflammatory and apoptotic factors, and related proteins were commonly detected in 16 studies. Besides, there were 16 studies focused on the detection of the myelin sheath, axon, and Schwann cells of the sciatic nerve, and 15 studies focused on the muscle appearance (measurement of muscle diameter and atrophy). Additionally, there were 7 studies that paid attention to the testing of enzymes and related peptides, but only one study had done the observation on the bone mineral density and blood vessel density tests. The detailed description of the molecules or factors involved are displayed in Figure 6.

3.5. Observation on the Mechanism of SNI with Acupuncture. According to the summary of the research in the past twelve years, the current speculated relevant mechanisms of SNI with acupuncture may include the following: (1) acupuncture could promote nerve regeneration and inhibit cell apoptosis by promoting the expression of nerve growth factor in SNI [22-24]; (2) acupuncture could reduce the expression of inflammatory factors and increase the content of related proteins to promote the recovery of SNI $[25,26]$; and (3) acupuncture could promote the proliferation of Schwann cells to the injured sciatic nerve to protect the nerve [27-29]. However, the research on the mechanism was mostly scattered, and they still could not represent the exact mechanism of acupuncture for SNI. 


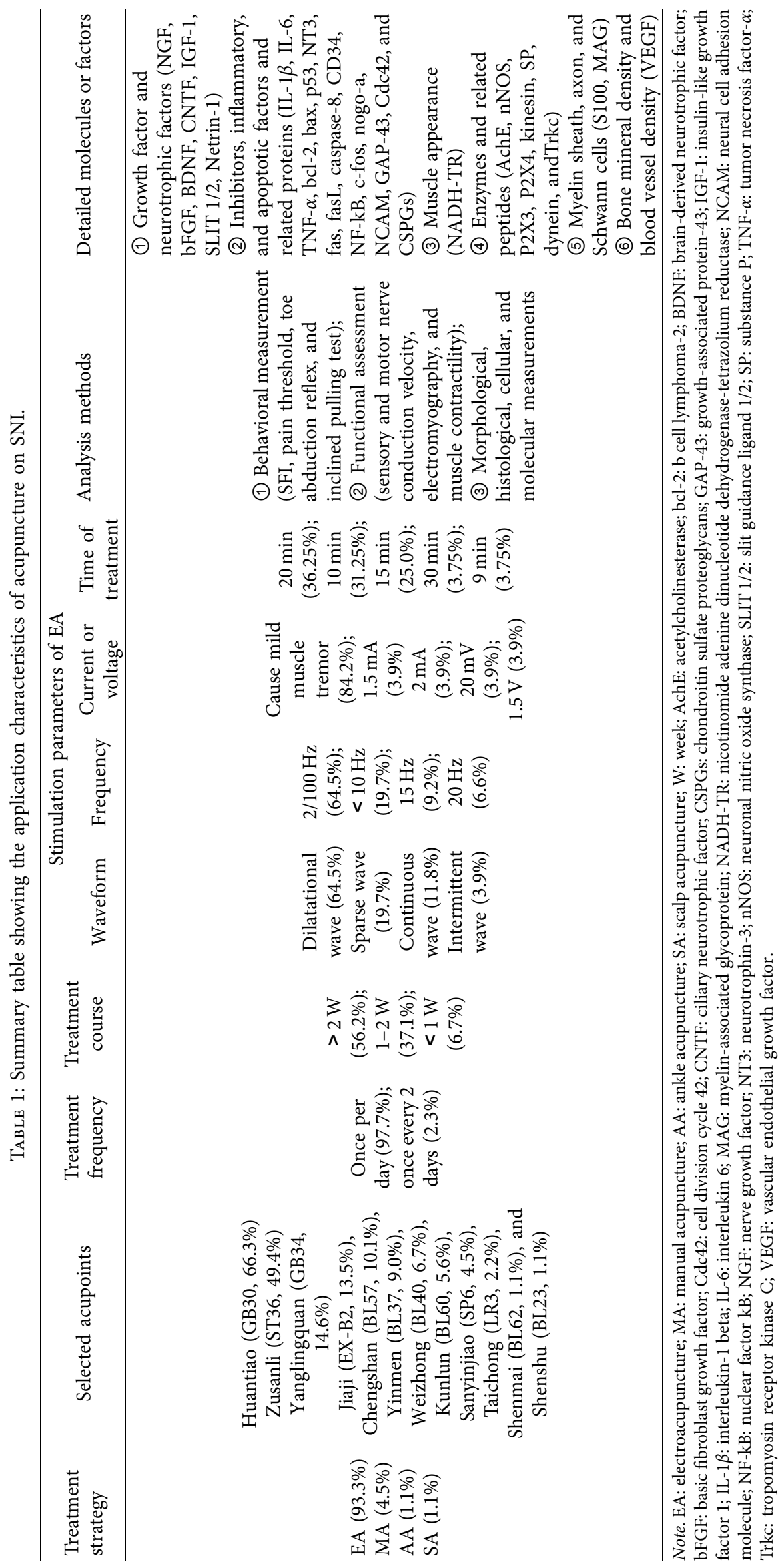




\section{Discussion}

In this study, the data about the experimental research of SNI with acupuncture has been collected from 2009 to 2020 and summarized in the items of animal model of SNI, type of interventions, selection of acupoints, course of treatment and its frequency, as well as approaches of assessment (Table 1). During the past twelve years, research in this field has made marked progress, step by step, to understand the underlying mechanisms of why acupuncture can play an effective role in alleviating SNI.

It is well known that, from bench to bed, experimental research is an indispensable way to promote the development of clinical progress. Although animal models with different types of SNI have been introduced to experimental research, such as transverse, ligation, clamp, traction, freezing, or drug injuries to the sciatic nerve, it is still difficult to select an appropriate one for mimicking the clinical patients. Among these models, the model with clamp injury is widely used to evaluate the effect of acupuncture on neural recovery $[15,17,19,30]$, and the model with transverse injury is mainly applied to assess the effect of acupuncture on neural regeneration [31]. Besides these two types, the model with ligation injury is also used; however, it seems difficult to control the tightness of the ligation in unity $[20,32]$. For the model with traction injury, it is also difficult to control the elongation of the sciatic nerve with two forceps between both ends of the nerve [33]. Additionally, the model with freezing injury is also applied. The problem is that the cryocooling probe not only damages the nerve, but also involves its surrounding tissues [33]. Therefore, considering the advantages and disadvantages of these animal models, the model with clamp injury is more suitable for investigating the underlying mechanism of acupuncture for meliorating the SNI.

In terms of the interventions for the SNI, electroacupuncture was recommended according to the collected data, which is consistent with the situation in clinical treatment. The possible reason might be that the parameters of stimulation with electroacupuncture can be controlled as demands on its strength and frequency [34]. Indeed, numerous studies have demonstrated that electroacupuncture has a good analgesic effect on various kinds of painful diseases [35, 36]. Here, it should be noted that although the $2 / 100 \mathrm{~Hz}$ dilatational wave is an appropriate choice for the animal models $[37,38]$ since the body size is different between small animals and humans, whether this parameter is suitable for the patients remains unanswered.

For the selection of acupoints, it is clear that, besides Jiaji (EX-B2) on the back, the other twelve acupoints are located closely or along the pathway of the sciatic nerve including its main trunk and its branches of the tibial, common peroneal, and sural nerves [39]. Traditionally, these acupoints belong to the meridians of the gallbladder (GB), stomach (ST), bladder (BL), spleen (SP), and liver (LR), respectively, and are orderly arranged along the longitudinal axis of the hind limb [39]. Although GB30 and ST36 are used most frequently, most acupoints belong to the bladder meridian including BL23, BL37, BL40, BL57, BL60, and BL62. Coincidently, the course of the bladder meridian as the traditional description is similar to the pathway of the sciatic nerve [39]. According to the spatial correlation between these acupoints and the sciatic nerve, they are the adjacent points to the sciatic nerve or its branches. In the clinical treatment of SNI, these acupoints can be used individually or together.

Although acupuncture treatment has obtained satisfactory effects on patients with SNI, due to the limitation of clinical studies, these effects are mainly evaluated on the variation of clinical symptoms and are seldom involved in their underlying mechanism [12-14]. As a comparison, experimental research has its own advantages in obtaining much more biological information from the perspective of pathologic histology. According to our collected data, most studies on the SNI with acupuncture were concentrated on neural protection and neural regeneration by way of antiinflammation and antiapoptosis [22-27]. These studies suggested that acupuncture could promote nerve regeneration, inhibit cell apoptosis, reduce the expression of inflammatory factors, improve the microenvironment of the injured area, and promote the proliferation of Schwann cells to repair the injured sciatic nerve [22-27]. However, there is still an absence of high-quality studies in this field. Therefore, the definite mechanism associated with acupuncture treatment on the SNI still needs further investigation in the future. Recently, there has been a new trend in SNI studies. Some researchers have paid attention to the SNI from the peripheral nervous system to the central nervous system, including the sensory input to the spinal dorsal horns and motor output from the spinal ventral horns $[25,26,40]$. It might be a new direction for acupuncture research on the SNI in the future.

\section{Conclusion}

In summary, the experimental research on the SNI with acupuncture has made marked progress in recent years, providing rich evidence for insight into the underlying mechanisms of acupuncture and how to alleviate the SNI. These experimental data are not only beneficial to future studies in this field but also for selecting appropriate acupoints and stimulating styles as clinical demands with SNI.

\section{Data Availability}

The datasets used for the current study are available from the corresponding author upon request.

\section{Conflicts of Interest}

The authors declare that they have no conflicts of interest.

\section{Authors' Contributions}

The article presented here was carried out in collaboration between all the authors. Hui Wang, Jia Wang, Jingjing Cui, Shitong Zhao, Dongsheng $\mathrm{Xu}$, and Shuang Wu searched the literature and analyzed the data. Jia Wang and Waizhu Bai designed the study. Hui Wang, Jia Wang, and Wanzhu Bai wrote the article. All authors read and approved the final version of the manuscript. 


\section{Acknowledgments}

This study was supported by the Project of National Key R\&D Program of China, No. 2019YFC1709103; the Scientific and Technological Innovation Project of China Academy of Chinese Medicine Sciences, No. CI2021A03407; National Natural Science Foundation of China (Nos. 82004492, 81774432, 81774211, and 81801561); and the Fundamental Research Funds for the Central Public Welfare Research Institutes (Nos. ZZ14-YQ-032, ZZ13-YQ-068, ZZ14-YQ034, ZZ201914001, ZZ202017006, and ZZ202017015).

\section{References}

[1] T. Gordon and G. H. Borschel, "The use of the rat as a model for studying peripheral nerve regeneration and sprouting after complete and partial nerve injuries," Experimental Neurology, vol. 287, pp. 331-347, 2017.

[2] L. E. Savastano, S. R. Laurito, M. R. Fitt, J. A. Rasmussen, V. Gonzalez Polo, and S. I. Patterson, "Sciatic nerve injury: a simple and subtle model for investigating many aspects of nervous system damage and recovery," Journal of Neuroscience Methods, vol. 227, pp. 166-180, 2014.

[3] S. Geuna, "The sciatic nerve injury model in pre-clinical research," Journal of Neuroscience Methods, vol. 243, pp. 39-46, 2015.

[4] X. Tao and T. M. Ma, "Development of researches on acupuncture treatment of peripheral nerve injury," Zhen Ci Yan Jiu, vol. 41, no. 1, pp. 90-93, 2016.

[5] C. C. Diogo, J. A. Camassa, J. E. Pereira et al., "The use of sheep as a model for studying peripheral nerve regeneration following nerve injury: review of the literature," Neurological Research, vol. 39, no. 10, pp. 926-939, 2017.

[6] J. A. Welch, "Peripheral nerve injury," Seminars In Veterinary Medicine and Surgery, vol. 11, pp. 273-284, 1996.

[7] D. D. Guo, X. G. Lu, X. L. Xu et al., "Therapeutic effect of vinorine on sciatic nerve injured rat," Neurochemical Research, vol. 43, no. 2, pp. 375-386, 2018.

[8] D. Grinsell and C. P. Keating, "Peripheral nerve reconstruction after injury: a review of clinical and experimental therapies," BioMed Research International, vol. 2014, Article ID 698256, 13 pages, 2014.

[9] M. Siemionow and G. Brzezicki, "Chapter 8 current techniques and concepts in peripheral nerve repair," International Review of Neurobiology, vol. 87, pp. 141-172, C, 2009.

[10] S. Sayad-Fathi, E. Nasiri, and A. Zaminy, "Advances in stem cell treatment for sciatic nerve injury," Expert Opinion on Biological Therapy, vol. 19, no. 4, pp. 301-311, 2019.

[11] L. L. Sasso, L. G. De Souza, C. E. Girasol, A. M. Marcolino, and R. R. De Jesus Guirro, "Photobiomodulation in sciatic nerve crush injuries in rodents: a systematic review of the literature and perspectives for clinical treatment," Journal of Lasers in Medical Sciences, vol. 11, no. 3, pp. 332-344, 2020.

[12] J. B. Tian, "Clinical observation of electroacupuncture in the treatment of sciatica caused by protrusion," Chinese Journal of Ethnomedicine and Ethnopharmacy, vol. 27, no. 4, pp. 105-106, 2018.

[13] Y. W. Li and F. Y. Men, "Electroacupuncture in the treatment of primary sciatica," Zhen Jiu Lin Chuang Za Zhi, vol. 27, no. 9, pp. 28-29, 2011.

[14] J. Wang, M. J. Wen, and D. Q. Zhou, "Recovery mechanism and therapeutic effect analysis of sciatic nerve injury," Chinese
Journal of Practical Nervous Diseases, vol. 16, no. 19, pp. 53-54, 2013.

[15] Y. Wang, Q. Tang, L. W. Zhu et al., "Effects of treatment of treadmill combined with electro-acupuncture on tibia bone mass and substance $\mathrm{P}$ expression of rabbits with sciatic nerve injury," PLoS One, vol. 11, no. 11, Article ID e0164652, 2016.

[16] L. H. Zeng, Y. Cen, J. J. Chen et al., "Effects of electroacupuncture on functional indices and pS6 expression following acute sciatic nerve crush injury in rats," Acupuncture in Medicine, vol. 38, no. 3, pp. 181-187, 2020.

[17] Y. Wang, C. F. Dong, R. N. Xu, Z. N. Guo, L. L. Zheng, and Y. M. Yuan, "Effect of electroacupuncture at Jiaji" (EX-B2) points combined with nerve mobilization on motor function and mRNA and protein expression of RhoA in rabbits with sciatic nerve injury," Zhongguo Zhen Jiu, vol. 39, no. 6, pp. 625-631, 2019.

[18] X. T. Liu, X. Tao, T. M. Ma et al., "The effect of electroacupuncture at different tissues of Huantiao" point on the phosphorylation expression of JNK and c-jun in the spinal cord of rats with sciatic nerve injury," Zhen Ci Yan Jiu, vol. 40, no. 5, pp. 373-377, 2015.

[19] Y. L. Liu, Y. Li, L. Ren et al., "Repair effect of deep acupuncture on Huantiao" point of sciatic nerve injury in rats," Zhen $\mathrm{Ci}$ Yan Jiu, vol. 39, no. 2, pp. 93-99, 2014.

[20] S. S. Li, W. Z. Tu, C. Q. Jia et al., "KCC2-GABAA pathway correlates with the analgesic effect of electro-acupuncture in CCI rats," Molecular Medicine Reports, vol. 17, no. 5, pp. 6961-6968, 2018.

[21] J. Yu, M. Wang, J. Liu, X. Zhang, and S. Yang, "Effect of electroacupuncture on the expression of agrin and acetylcholine receptor subtypes in rats with tibialis anterior muscular atrophy induced by sciatic nerve injection injury," Acupuncture in Medicine, vol. 35, no. 4, pp. 268-275, 2017.

[22] X. C. Ye, S. J. Shao, H. D. Guo, X. J. Han, Y. P. Liu, and P. P. Li, "Effect of electroacupuncture on brain derived neurotrophic factor of rats with sciatic nerve injury," Chinese Journal of Information on Traditional Chinese Medicine, vol. 24, no. 6, pp. 60-63, 2017.

[23] X. Du, R. H. Wang, P. L. Wang et al., "Effects of electroacupuncture on receptor DCC of nerve growth guidance factor netrin-1 of rats after sciatic nerve injury," Chinese Medicine Modern Distance Education of China, vol. 15, no. 5, pp. 128-130, 2017.

[24] Y. Yu, J. C. Wu, X. Q. Li et al., "Investigation the effect of electro-acupuncture on NT-3 expression in ventricolumna of sciatic nerve injury rats," Global Traditional Chinese Medicine, vol. 7, no. 4, pp. 262-265, 2014.

[25] X. Q. Li, T. Y. Yu, Y. Yu et al., "Effects of electroacupuncture on serum myelin basic protein and its antibody in rats with sciatic nerve injury," Journal of Beijing University of Traditional Chinese Medicine, vol. 38, no. 12, pp. 842-846, 2015.

[26] H. Chen, M. Y. Huang, W. Y. Yu, D. Lin, and L. L. Lin, "After effect of electroacupuncture at Jiaji acupoint on the protein expression of P2X4, P2X7 in chronic constriction injury rats," Journal of Rehabilitation, vol. 28, no. 1, pp. 37-40, 2018.

[27] X. Du, R. H. Wang, Q. H. Zhang, and W. Hu, "Effects of electroacupuncture on Schwann cells and nerve function in rats with sciatic nerve injury," Shaanxi Journal of Traditional Chinese Medicine, vol. 33, no. 5, pp. 621-624, 2012.

[28] R. H. Wang, J. Y. Dai, H. Y. Qu, Y. W. Yu, and X. Du, "Effect of acupuncture on Schwann cells after sciatic nerve injury," Journal of Shaanxi University of Chinese Medicine, vol. 2, pp. 30-31, 2008. 
[29] S. J. Shao, B. Z. Shan, J. H. Zhang, Y. X. Zhao, A. S. Yu, and Z. G. Yan, "Effect of electroacupuncture serum on Schwann cell proliferation," Shanghai Journal of Acupuncture and Moxibustion, vol. S1, pp. 58-60+92, 2000.

[30] N. S. Hoang, C. Sar, J. Valmier, V. Sieso, and F. Scamps, "Electro-acupuncture on functional peripheral nerve regeneration in mice: a behavioural study," BMC Complementary and Alternative Medicine, vol. 12, p. 141, 2012.

[31] J. J. Wu, Y. C. Lu, X. Y. Hua, S. J. Ma, and J. G. Xu, “A longitudinal mapping study on cortical plasticity of peripheral nerve injury treated by direct anastomosis and electroacupuncture in rats," World Neurosurg, vol. 114, pp. e267e282, 2018.

[32] D. D. Zhao, C. L. Tang, S. Q. Huang et al., "Effect of electroacupuncture on amyotrophia and expression of myogenic differentiation-related genes of gastrocnemius in rats with chronic constriction injury of sciatic nerve," Zhen Ci Yan Jiu, vol. 44, no. 1, pp. 37-42, 2019.

[33] J. Su, C. Y. Ling, and L. M. Lei, "An overview of modeling and evaluation indexes of experimental animals with sciatic nerve injury," Chinese Journal of Tissue Engineering Research, vol. 25, no. 26, pp. 4186-4191, 2021.

[34] T. Taguchi and R. Taguchi, "Effect of varying frequency and duration of electroacupuncture stimulation on carrageenaninduced hyperalgesia," Acupuncture in Medicine, vol. 25, no. 3, pp. 80-86, 2007.

[35] J. T. Kong, C. Puetz, L. Tian et al., "Effect of electroacupuncture vs sham treatment on change in pain severity among adults with chronic low back pain: a randomized clinical trial," JAMA Network Open, vol. 3, no. 10, Article ID e2022787, 2020.

[36] Q. M. Hu, X. L. Zheng, X. J. Li et al., "Electroacupuncture alleviates mechanical allodynia in a rat model of complex regional pain syndrome Type-I via suppressing spinal CXCL12/CXCR4 signaling," The Journal of Pain, vol. 21, no. 9-10, pp. 1060-1074, 2020.

[37] K. M. Shin, I. G. Ko, S. E. Kim et al., "Low-frequency electroacupncture improves locomotor function after sciatic crushed nerve injury in rats," J Exerc Rehabil, vol. 14, no. 6, pp. 927-933, 2018.

[38] Q. X. He, S. T. Pan, Y. R. Chen, and T. M. Ma, "Lower frequency electroacupuncture is better in promoting recovery of limb locomotion in rats with sciatic nerve injury by reducing local inflammatory reaction," Zhen Ci Yan Jiu, vol. 44, no. 8, pp. 571-576, 2019.

[39] X. Y. Shen, Meridians and Acupoints, Chinese Medicine Press, Beijing, China, 2nd edition, 2010.

[40] S. S. Li, W. Z. Tu, X. Jiang et al., "Effect of electro-acupuncture at Zusanli (ST36) and Yanglingquan (GB34) acupoint on expression of TrkB receptor in Spinal of CCI rats," Chinese Archives of Traditional Chinese Medicine, vol. 35, no. 11, pp. 2878-2881, 2017. 